\title{
L'Hôpital général de Genève de 1780 à 1798 : quelques indices de médicalisation
}

Micheline Louis-Courvoisier

\section{SUMMARY}

The medicalization of a hospital can be considered in several ways. This study covers the two last decades of the 18th century of the General Hospital of Geneva. In a first part it analyses the space alotted to sick and healthy people, respectively. The internal organization of the hospital discloses the criteria used for selection. The second part analyses the population of patients with respect to age, duration of hospitalization and, if recorded in a meaningful way, the diseases. The majority of patients were under 30 years old and had a stay of less than 30 days. A third aspect of medicalization studied is the role played by various groups of personnel. It shows that the administrators allowed but a limited autonomy to the physicians and surgeons, yet tried to hire high-ranking medical personnel which in turn contributed to the modernization of the hospital.

\section{RÉsumé}

Les indices de médicalisation d'un hôpital peuvent être appréhendés par plusieurs biais. Cette étude, qui couvre les deux dernières décennies du XVIIIe siècle, se propose d'analyser en premier lieu la place dont disposent les malades au sein de l'Hôpital général de Genève, par rapport aux pensionnaires valides. L'organisation intérieure de l'institution permet de révéler quels étaient les critères de répartition. Une deuxième partie est consacrée aux malades euxmêmes, contenant des indications sur l'âge, la durée de séjour des malades, et dans la mesure du possible, les affections qui règnent dans l'établissement. On constate qu'une forte proportion de malades a moins de 30 ans et effectue un séjour hospitalier de moins d'un mois. Le dernier aspect de médicalisation étudié ici tend à évaluer le rôle joué par le corps médical, principalement par les médecins et les chirurgiens. Cette partie révèle que si les administrateurs ne laissent pas une grande place aux médecins et aux chirurgiens, en revanche, ils engagent des personnalités médicales d'envergure qui, par leur présence, contribuent à une certaine modernisation. 
Le concept de médicalisation d'un hôpital englobe divers aspects, indépendants les uns des autres, aspects qui sont plus ou moins développés dans les hôpitaux de l'Ancien Régime; il s'agit donc en premier lieu d'essayer de définir ce concept ${ }^{1}$.

L'analyse des conditions d'hospitalisation et des hospitalisés représente un moyen essentiel d'approcher le niveau de médicalisation d'un hôpital ${ }^{2}$. En effet, les conditions d'admission sont importantes à déterminer, dans la mesure où elles permettent de savoir si les hospitalisés étaient admis par un médecin ou un chirurgien, où s'ils étaient acceptés par un administrateur. D'autre part, l'étude des hospitalisés nous renseigne sur l'importance accordée par les directeurs aux malades, et quelle place leur était accordée au sein de l'établissement. En effet, si «la médicalisation substitue peu à peu à une population de nécessiteux une population de malades, qui requiert des soins médico-chirurgicaux bien définis» ${ }^{3}$, il convient de cerner en quoi consiste cette population médicale. Dans cette même problématique, il est aussi important d'évaluer au sein de l'institution le degré de séparation qui existait entre les malades, les nécessiteux, les orphelins, les vieillards ${ }^{4}$.

Déterminer si une institution hospitalière constitue un lieu de formation pour le personnel médical représente un autre indice de médicalisation ${ }^{5}$. Il s'agit là d'établir si l'hôpital en question fournit une structure adéquate pour l'enseignement, s'il contient un nombre de lits suffisant pour servir de lieu d'expérimentation, ou encore s'il offre des possibilités d'effectuer des dissections. Cette question va de pair avec la volonté d'améliorer les soins administrés aux malades hospitalisés, volonté dont la dynamique est contrôlée par les administrateurs. Cette approche implique également l'étude du personnel soignant, dont le rôle dans l'établissement est à évaluer si l'on veut en définir le niveau de médicalisation ${ }^{6}$. En effet, parfois une mentalité médiévale de certaines sœurs hospitalières, basée essentiellement sur le secours spirituel, pouvait entraver les progrès médicaux et faire obstacle aux volontés de modernisation des médecins ou chirurgiens, ou des administrateurs? ${ }^{7}$.

Un autre aspect de la médicalisation d'un établissement, beaucoup plus difficile à mettre en lumière, consiste à évaluer les motivations des administrateurs lorsqu'ils décident d'accepter un malade. Cette question se pose dans la mesure où la charité ancienne n'opérait pas de distinction «entre détresse morale ou matérielle et détresse physiologique» ${ }^{8}$. Dans cette optique, un hôpital médicalisé met l'accent sur la maladie et non sur le besoin de la population hospitalisée ${ }^{9}$. 
En résumé, le terme de médicalisation recouvre plusieurs attributs. Premièrement, il signifie, quand il qualifie un hôpital, lieu de formation pour médecins et chirurgiens. Deuxièmement, il indique une nette séparation entre malades et valides, avec un accent mis sur les soins des malades et sur la qualité du personnel soignant. Troisièmement, il révèle une certaine détermination des autorités à soigner la maladie plus que la pauvreté.

Pendant longtemps, on a décrit les hôpitaux de l'Ancien Régime essentiellement comme des mouroirs, des prisons pour vagabonds et mendiants, ou au mieux, une institution uniquement destinée à secourir les pauvres méritants. Cependant, si les institutions hospitalières avaient effectivement cette fonction à caractère social, il n'en demeure pas moins qu'elles avaient également un rôle médical à jouer dans une cité, quelle que soit la destination première de l'établissement. Nous faisons ici allusion à la classification de Muriel Joerger, qui a répertorié trois catégories d'hôpitaux: 1) les hôpitaux qui garantissaient une assistance sous toutes ses formes dans une paroisse, assurant une distribution de vivres et de vêtements et recueillant les malades, les enfants, les incurables, les invalides; 2) les hôtels-Dieu, réservés aux malades, excluant les incurables, et parfois les aliénés, les vénériens, les galeux ou les femmes en couches; 3) les hôpitaux généraux, destinés à «renfermer» les vagabonds, à accueillir les malades dont les hôtelsDieu ne voulaient pas ${ }^{10}$.

Chaque hôpital connaît sa propre évolution et, par conséquent, la généralisation dans ce domaine peut mener à l'erreur. Les hôpitaux de différentes villes européennes se développèrent de manières très différentes; Sandra Cavallo ${ }^{11}$, par exemple, constate une grande continuité dans le développement de deux hôpitaux turinois, destinés aux malades depuis leur création en 1440 et 1575, dans le domaine de l'expérimentation, de la pratique médicale et de l'enseignement, tandis qu'Eduard Seidler, au contraire, propose une réelle rupture entre l'hôpital du XVIIIe siècle et ses prédécesseurs, qui passa du refuge à l'institution pour malades ${ }^{12}$. Par ailleurs, les hôpitaux florentins connurent une grande tradition hospitalière, et furent admirés par toute l'Europe. Ils employaient notamment des médecins et des chirurgiens choisis parmi les six meilleurs de la cité ${ }^{13}$. En revanche, l'hôtelDieu de Nîmes, destiné essentiellement aux malades, jouait plutôt le rôle de relais pour de jeunes travailleurs de passage; sa population était saine et vigoureuse et un seul apprenti-chirurgien vivait sur place au XVIIIe siècle ${ }^{14}$.

Dans les paragraphes suivants, je vais m'attacher à analyser de plus près les indices de médicalisation de l'Hôpital général de Genève, durant les deux 
dernières décennies de la République genevoise, jusqu'à 1798, date de l'occupation française. J'essaierai également de déterminer si une évolution de la médicalisation voit le jour durant cette fin de siècle.

\section{Bref historique}

L'Hôpital général genevois fut créé en 1535, quelques mois avant la Réforme, mais après l'abolition de la messe; ce détail a son importance, car les catholiques de l'époque qui désiraient rester fidèles à leur confession durent quitter la ville; or, après leur départ, les Conseils décidèrent de confisquer leurs biens et attribuèrent une partie de ces ressources au nouvel Hôpital. Cette institution était destinée «tam pour les povres petits enfants orphelins trouvés, femmes vefves, gens anciens, povres filles et toute aultres manieres de gens tam estrangiers que privés de toutes nations venants respayre ${ }^{15}$ audit hospital general» ${ }^{16}$. Sa fonction consistait donc à distribuer de la nourriture (du pain essentiellement) et des vêtements aux nécessiteux de la cité, recueillir les orphelins, les vieillards sans famille, les étrangers de passage et les malades. Elle était administrée par quatre procureurs, qui tous appartenaient aux différentes instances politiques de la cité; leurs ordres étaient exécutés par l'hospitalier, qui habitait avec sa famille dans l'institution et qui exerçait, aidé de sa femme, le rôle de gérant de la maison. L'hospitalier devait s'occuper de tout, aussi bien des différentes productions effectuées par les gens bien portants de la «Maison», que de la distribution de pain aux assistés de la ville, que des malades en pension dans l'Hôpital; il représentait la clé de voûte de l'institution. Cette structure administrative, décrite de manière très sommaire, resta la même pendant plus de trois siècles, à l'exception du nombre de procureurs qui augmenta au gré de la croissance de la cité et de la demande; à l'époque qui nous intéresse, huit hommes siégeaient au sein de cette administration hospitalière, sous l'égide d'un syndic et d'un conseiller ${ }^{17}$.

Une de ses spécificités consistait dans le fait qu'il résidait dans une très petite république; c'était donc sous un seul toit et une seule administration qu'il rassemblait ses différentes fonctions, à l'égard des malades ou des bien portants, des aliénés ou des mères célibataires, des orphelins ou des vieillards. La taille de l'Etat dans lequel s'inscrivait une institution hospitalière n'est pas seulement importante pour expliquer son organisation intérieure ou sa grandeur, mais elle détermine également le souci et la responsabilité 
des autorités vis-à-vis des pauvres et des malades de la cité. Si nous comparons avec Lausanne, l'établissement hospitalier vaudois était beaucoup moins développé, malgré les efforts du célèbre médecin Tissot, en raison de l'inertie des autorités municipales, qui avaient tendance à se reposer sur les Bernois ${ }^{18}$.

L'aspect médical de l'Hôpital genevois était présent dès sa création et se manifesta par la présence d'un chirurgien, engagé par l'institution pour soigner les assistés de la ville et les pensionnaires de la maison; il fut rapidement rejoint par un médecin qui entra en fonction en $1558^{19}$. Cette préoccupation, même minime, de l'intérêt des malades demeura constante au cours des siècles qui suivirent, et reste à démontrer; je n'en donnerai ici que trois exemples illustratifs : le premier concerne la construction du nouvel Hôpital, entre 1707 et 1712; en effet, au cours des débats qui élaborèrent la conception du nouveau bâtiment, en 1699, le gouvernement enjoignit les procureurs de «mettre en état quelque apartement convenable pour y placer les malades, en sorte que chacun puisse avoir son lict séparé» ${ }^{20}$, le manque de lits ne permettant pas de secourir les malades de manière satisfaisante. Il est probable qu'aux moments de grande affluence, ce souhait n'ait pas toujours été réalisé, mais il n'en demeure pas moins que cette mention nous indique une certaine précocité dans la conception médicale des autorités et des procureurs. Un autre indice de médicalisation frappant date de 1746, année durant laquelle Antoine Sabourin, le chirurgien de l'Hôpital, demanda aux administrateurs et au Conseil ordinaire la permission de pouvoir disséquer des cadavres de femmes, afin de contribuer à l'instruction des accoucheuses $^{21}$; il considérait donc l'Hôpital comme un lieu de formation et d'enseignement. On ne sait pas si ces cours eurent longue vie, mais la volonté de les donner représente déjà un indice de médicalisation. Le troisième exemple date de la même année, durant laquelle l'hospitalier von der Strassen reçut l'ordre de recevoir tous ceux qui seraient amenés à l'Hôpital avec un bras ou une jambe cassés, sans avoir obtenu au préalable l'accord d'un des procureurs $^{22}$. Cela indique une sorte de procédure d'urgence pour les blessés dont l'état exigeait un secours médical. Il est clair que ces indices sont ponctuels et ne permettent pas d'affirmer que l'objectif de l'administration avait un caractère médical plutôt que social, ce qui serait erroné; cependant, ils nous montrent une préoccupation des autorités concernant les soins médicaux.

A l'époque qui nous intéresse, l'établissement se dressait au Bourg-deFour, à l'emplacement de l'actuel Palais de Justice, administré par les huit procureurs et gouverné par l'hospitalier. L'institution vivait alors dans la 
tourmente politique et sociale des ultimes années de l'Ancien Régime, mais n'en ressentit pas trop les effets, excepté la surcharge souvent excessive due à l'afflux de soldats dans la ville.

\section{Organisation intérieure de l'Hôpital}

L'organisation du bâtiment nous permet de rendre l'Hôpital plus concret et de déterminer quels espaces étaient réservés aux malades; les inventaires nous révèlent aussi les quelques objets pouvant servir aux soins des malades.

Du point de vue immobilier, deux bâtiments constituaient l'institution hospitalière: le bâtiment principal du Bourg-de-Four, et la maison de la Discipline, qui devint plus tard la prison Saint-Antoine et qui servait de maison de correction ${ }^{23}$, mais également d'asile pour les aliénés ${ }^{24}$.

La bâtiment principal comportait trois étages; le rez-de-chaussée était réservé à la fabrication et la distribution du pain dans l'aile droite, à l'administration, avec une chambre pour les assemblées bi-hebdomadaires des procureurs, une chambre pour le secrétaire et une autre pour les archives, de même que le temple; quelques chambres y étaient également destinées à recevoir les passants. L'appartement de l'hospitalier se situait au premier étage, côtoyant ainsi la partie réservée aux hommes, malades et sains, qui se répartissaient les différentes chambres de l'étage. Au deuxième logeaient les femmes, au-dessus desquelles se trouvaient des greniers qui parfois servirent à héberger des soldats. Attenant à ce bâtiment se dressait un autre corps de logis, qui comportait également trois étages, chacun d'eux étant subdivisés en trois grandes pièces d'égale grandeur, avec au rez la cuisine et deux réfectoires, les hommes au premier et les femmes au deuxième ${ }^{25}$.

Jusqu'en 1789, les malades étaient situés dans deux chambres du bâtiment principal, dans l'aile droite, au-dessus de l'espace réservé à la fabrication du pain; les hommes et les femmes disposaient d'une grande salle contenant vingt lits ${ }^{26}$ de fer garnis d'étoffe verte et d'une plus petite chambre dotée de six lits de noyer ${ }^{27}$. Ajoutons que quatre lits par étage étaient réservés aux gouverneurs et gouvernantes, qui dormaient avec les hospitalisés; par conséquent, 22 lits étaient destinés à recevoir des malades de chaque sexe. Selon un rapport de $1789^{28}$, la moyenne de malades se situait à 18 hommes et 19 femmes, mais leur nombre pouvait s'élever occasionnellement à 33 hommes et 24 femmes. D'après les auteurs de ce rapport, il était temps de déplacer les hommes dans des chambres plus adéquates et plus 
spacieuses. Plusieurs de leurs arguments étaient déterminés par l'aspect médical de la situation des hospitalisés du sexe masculin; ils évoquaient d'abord une meilleure aération dans les trois salles appartenant au plus petit corps de logis attenant au bâtiment principal; en effet, à la fin du XVIIIe siècle, on accordait une grande importance à la hauteur des plafonds et aux dimensions des fenêtres, considérant qu'une bonne aération ralentissait la vicissitude de l'air exhalé par les malades. Ils se rendaient également compte que trois chambres parallèles permettaient une meilleure répartition des malades selon leurs affections; en outre, ces chambres étaient situées près de la cour de la Discipline, qui constituerait un promenoir précieux pour les convalescents; mais surtout, cela mettrait à disposition un plus grand nombre de lits, dont le total monterait à 48 lits pour les hommes. Les membres de la commission qui rédigèrent ce rapport furent suivis par tous les directeurs de l'Hôpital, et les hommes malades furent transportés dans l'autre corps de logis.

La situation des femmes était légèrement différente, malgré le fait que leur localisation à l'intérieur de l'Hôpital fut identique à celle des hommes. En effet, les rédacteurs du rapport soulignaient les mêmes avantages concernant le déplacement des femmes, cependant, ils mettaient en garde les procureurs, afin qu'ils évitent soigneusement d'assigner plus de trente lits aux femmes malades car «on a trop senti leur disposition à y demeurer lors même que les secours de la médecine leur sont inutiles» ${ }^{29}$. Elles déménagèrent finalement une année plus tard, mais on ne sait malheureusement pas de combien de lits elles disposèrent exactement ${ }^{30}$.

Ajoutons encore que les vénériens n'étaient pas avec les autres malades, mais logeaient à l'entresol, dans la chambre dite «noble» qui comportait six lits de bois en $1772^{31}$. En 1786, on leur attribua une chambre contiguë pour y mettre les vénériens en fin de traitement, afin d'éviter le mauvais air causé par le «frottage» des véroles ${ }^{32}$, et en 1799 , on assembla les vénériens et les galeux $^{33}$. Etait-ce le début d'un «service de dermatologie», pour employer un terme anachronique?

Une évolution se fit entre 1772 et $1825^{34}$, et plus probablement après le tournant du siècle si l'on considère les espaces réservés aux malades; en effet, en 1825, on constate une plus grande séparation entre les différentes catégories de malades hospitalisés: à cette date, il existait une chambre pour les hommes blessés et une autre pour les fiévreux, tandis qu'au deuxième étage, les femmes semblaient indistinctement placées dans les trois salles qui leur étaient destinées, exception faite pour les femmes souffrant de maladies de la 
peau et d'affections vénériennes, qui étaient logées dans une chambre à part, composée de cinq lits. En outre, la Discipline abritait les gangreneux, les hommes vénériens et galeux au premier étage, les aliénés des deux sexes au second étage et les aliénés reclus au troisième ${ }^{35}$.

Il est à noter qu'aucun endroit n'était réservé aux actes médicaux de quelque nature qu'ils fussent. En effet, aucune mention de salle d'opération, d'auscultation, de cabinet ne figure même dans l'inventaire de 1825. C'est seulement sur les plans de P.Guillebaud, effectués en 1835, qu'apparurent des locaux à affectations médicales, comme la salle d'opération et la salle de dissection. En revanche, cette absence ne signifiait pas qu'aucun acte médical n'était effectué à l'Hôpital ou pour l'Hôpital; en effet, des opérations même lourdes avaient lieu dans l'institution, dans la chambre des malades, à l'instar de celle effectuée, en 1771, sur Jean Langin, qui subit une amputation de jambe suite à des gelures au pied ${ }^{36}$. D'autre part, il existait, de 1728 à 1733 en tous cas, une chambre d'anatomie dépendante de l'Hôpital, située dans le Petit Hôpital ${ }^{37}$, dans laquelle se donnait un cours public pour les chirurgiens de la ville, soit d'anatomie soit de chirurgie, cours donné par un chirurgien et présidé par un médecin ${ }^{38}$; nous ne savons pas ce qu'il advint de cette chambre d'anatomie.

Le matériel d'ordre médical trouvé dans les inventaires était bien rare. En 1772 , on ne répertorie que quelques seringues et quatre tabliers de chirurgien, que l'on trouvait déjà dans l'inventaire de $1757^{39}$. En revanche, en 1825, huit tabliers de médecin et 55 habits d'infirmiers étaient dans l'entrée de la salle des hommes malades; de même, 54 tabliers d'infirmières se trouvaient dans les salles des femmes malades ${ }^{40}$.

L'étude de l'Hôpital sous l'angle topographique nous montre simultanément l'évolution de sa médicalisation et l'immobilisme de cette institution. L'immobilisme était surtout caractérisé par la permanence des attributions des espaces en général: les hommes au premier étage, les femmes au second; cette séparation entre hommes et femmes est d'ailleurs encore actuelle. D'autre part, les malades cotôyèrent les pensionnaires bien portants, jusque dans les années 1830 en tous cas: sa fonction resta sociale aussi bien que médicale. Cependant, au sein de cet immobilisme s'effectuait petit à petit une lente évolution; les malades, hommes et femmes, furent déplacés dans des chambres plus adéquates, afin qu'ils puissent être mieux soignés, soit en séparant les maladies soit en protégeant les convalescents, comme ce fut le cas pour les vénériens, soit encore en favorisant l'aération des chambres; cela préparait les changements effectués en 1825, qui permirent l'aménagement 
d'une salle pour les fiévreux et d'une salle pour les blessés, ébauches d'un service de médecine et d'un service de chirurgie. Toutefois, le matériel trouvé à l'Hôpital au cours de cette fin de siècle ne permet pas de constater une quelconque évolution, qui se produisit de façon plus accélérée pendant les deux premières décennies du siècle suivant.

\section{Les malades}

Les malades ${ }^{41}$ représentent une deuxième approche de la médicalisation de l'Hôpital genevois, et son évolution, malgré la difficulté que l'on rencontre pour obtenir des informations sur les hospitalisés. Il s'agit tout d'abord de définir les critères de la maladie, en dépit de la sécheresse des archives; pour plus de sécurité, je n'ai retenu que les hospitalisés qualifiés de «malades», ou «très malades», les «blessés», ou encore ceux qui moururent moins d'une semaine après leur hospitalisation, partant du principe qu'ils étaient probablement déjà malades lors de leur entrée.

La dernière décennie connut une évolution des admissions d'ordre médical, puisqu'en 1785, 34\% des entrées furent admises pour des raisons médicales, alors qu'en $1795,58 \%$ de la population hospitalière étaient malades. Tant que ces pourcentages ne seront pas inscrits dans une série quantitative beaucoup plus longue, on ne peut en conclure une évolution significative. Cependant, ils coïncident avec un discours visant également à la médicalisation des hospitalisés. C'est ainsi qu'Abraham Joly ${ }^{24}$, hospitalier très respecté de tous, se plaignit, en 1789 , que «la chambre des malades se remplissoit de gens peu interessans par leur yvrognerie et dont quelques uns n'ont pas un droit légitime à être reçus dans la Maison» ${ }^{43}$. En outre, dès 1784, la volonté de faire sortir «toutes les personnes qui sont en santé et inutile[s] $\gg^{44}$ existait déjà et on ajoutait qu'il ne fallait pas oublier les valides dans l'institution et encore, que l'on devait ne recevoir que des malades qui seraient renvoyés à leur guérison. Notons que jusqu'à présent c'est la première fois que nous lisons ce genre de discours dans les archives hospitalières, c'est pourquoi il est important de le souligner, même si dans la pratique il ne fut suivi que beaucoup plus tard. Il y avait un réel désir de médicaliser l'Hôpital en ne gardant que les malades, même si les motifs de cette modernisation étaient plutôt d'ordre économique. 
Les hommes malades étaient très légèrement majoritaires (50 \% en 1785 , $57 \%$ en 1790 et $52 \%$ en 1795) et il est important de constater que la plus forte proportion des hospitalisés avaient entre 20 et 29 ans. Cela rejoint ce que d'autres auteurs ont trouvé à Lyon, affirmant notamment que la jeunesse représentait une caractéristique constante des malades hospitalisés ${ }^{45}$.

Des enfants étaient également acceptés par l'Hôpital, même lorsqu'ils n'étaient pas orphelins: ainsi, Marie Guillou, six ans, effectua un séjour dans l'Hôpital, pour être soignée de son mal vénérien ${ }^{46}$, ou Jean-Pierre Paton, treize ans, hospitalisé pour une blessure suite à un coup de feu, doublée du tétanos; il mourut quinze jours plus tard ${ }^{47}$.

La durée de séjour est également révélatrice de la fonction qu'avait l'institution; en effet entre 42 et $65 \%$ des malades restèrent moins d'un mois dans l'institution et $38 \%$ effectuèrent un séjour de moins de 15 jours en 1795 . Là encore, on peut penser avec 0 . Faure et D. Dessertines que l'hôpital constituait un moyen de survie dans des moments de déstabilisation individuelle, mais n'était pas vécu comme une solution à long terme ${ }^{48}$. Jean Bertrand illustre bien ce propos: en 1788, il fit un séjour de 28 jours, après une chute; puis il revint à deux reprises en 1789 , pour 24 et 22 jours, «malade de misère et de froid» et «malade de misère et de vermine»; et en 1790, il fut à nouveau hospitalisé pour 10 jours, simplement pour maladie ${ }^{49}$. Cet homme vivait probablement à la limite de ses ressources matérielles et physiques et au moindre accroc son équilibre précaire s'effondrait.

Certains malades restaient plus longtemps, comme Louise Geoffrin, qui fut hospitalisée durant 153 jours avec un bras cassé et blessé ${ }^{50}$, ou Marie Mottu, qui reçut un coup de faux sur le pied et qui resta 120 jours, probablement dans la même chambre que Louise, puisque leurs accidents eurent lieu à la même époque ${ }^{51}$.

La curiosité m'a amenée à regarder de quoi souffraient les malades de l'Hôpital. Si le diagnostic n'a pas un rapport direct avec la médicalisation, il permet cependant de voir et peut-être, un jour, de comparer les différents termes utilisés par les médecins et les chirurgiens. D'autre part, en ce qui concerne l'accouchement, nous le verrons plus loin, il révèle peut-être une attitude nouvelle vis-à-vis de la naissance.

Toutefois, procéder à un diagnostic rétroactif a toujours représenté un exercice difficile, approximatif, voire dangereux. En effet, des nouveaux savoirs signifiaient de nouveaux noms et un ancien diagnostic pouvait englober plusieurs entités nouvelles; c'est ainsi que l'asthme renvoyait non 
seulement à une affection allergique, mais aussi à d'autres troubles respiratoires ayant une toute autre cause ${ }^{52}$. D'autre part, les médecins contemporains n'utilisaient pas toujours les termes dans le même sens; cela rend la tâche plus compliquée. En outre, la méfiance est de mise lorsqu'il s'agit de termes médicaux simples, comme la phtisie qui ne signifie pas forcément la tuberculose pulmonaire ${ }^{53}$; selon J. P. Peter, les termes les plus simples mais les plus fourbes sont ceux qui restent une entité nosologique actuelle, comme: les aphtes, angines, coliques, rougeole, grippe, lumbago, furoncle, oreillons, sciatiques, urticaire ${ }^{54}$. D'autre part, les médecins de l'époque étaient à cheval sur deux systèmes: les raisonnements savants s'estompaient peu à peu face à un regard basé sur l'expérience et l'observation; l'étude des symptômes et des syndromes cédèrent leur place à l'analyse des lésions ${ }^{55}$.

Pour les malades pris en considération dans cette étude, j'ai tenté de classer leurs maladies dans de très grandes catégories. Nous n'avons bien sûr pas le diagnostic pour chaque personne hospitalisée: entre 34 et $61 \%$ des hospitalisés sont qualifiés de «malade»; en revanche, le Livre des Morts ${ }^{56}$ a pu compléter nos indications dans le cas des malades morts à l'Hôpital.

Parmi les maladies les plus fréquentes, on retrouve les maladies vénériennes, les maladies «internes» ${ }^{57}$ et les maladies «dermatologiques» ${ }^{58}$. L'institution abritait également des blessés, des aliénés, des malades atteints de langueur, de marasme ou de mélancolie.

En 1795, il existe une très légère augmentation des accouchements à l'Hôpital, qui n'est peut-être que conjoncturelle, mais qui, cependant, coïncide avec un changement d'attitude vis-à-vis des femmes en couches. Auparavant, les femmes entraient à l'Hôpital une fois que leur situation maritale, en général peu claire ${ }^{59}$, était réglée avec la justice, et on pourrait presque dire que l'institution adoptait une attitude paternaliste à leur égard; en revanche, en 1795, les femmes restaient beaucoup moins longtemps et étaient plus nombreuses à venir mettre au monde leurs bébés dans l'Hôpital, même si ce n'était pas courant pour autant. Si l'on ajoute encore que certaines de ces femmes semblaient avoir une vie conjugale normale ${ }^{60}$, cela peut représenter une ébauche d'un nouveau comportement face à l'accouchement, qui fut renforcé quelques années plus tard par l'ouverture d'une maternité par le chirurgien Louis Jurine ${ }^{61}$, en 1808, sous l'impulsion du préfet. Il faut cependant souligner que l'accouchement s'effectuait toujours à domicile dans la grande majorité des cas.

La mortalité hospitalière variait entre 11,5 et $16 \%$ durant les trois années étudiées, taux qui n'est pas très éloigné de ce qu'ont trouvé 0 . Faure 
et D. Dessertine pour Lyon, à savoir un taux de mortalité de $11,5 \%$ en $1866^{62}$, soit presque un siècle plus tard. Mais là encore, nos chiffres ne sont qu'indicatifs; non seulement ils devraient être inclus dans une longue série pour montrer une tendance, mais il faudrait également étudier de près les classes d'âge les plus touchées par la mortalité hospitalière.

\section{Les médecins et chirurgiens de l'Hôpital}

Le rôle des médecins et des chirurgiens, bien que difficile à définir, représente un indice essentiel de la médicalisation de l'Hôpital. Il en est d'ailleurs de même pour l'importance accordée au personnel soignant: mais ce dernier n'aura malheureusement pas sa place dans cette étude, car les informations manquent pour l'instant. Cependant, nous pouvons tout de même souligner que la fin du XVIIIe siècle fut également un tournant pour la place accordée aux aides-soignants. Dans la terminologie déjà un changement s'amorçait, avec pour la première fois le terme d'«infirmier» utilisé en $1796^{63}$, qui cohabita encore plusieurs années avec celui de gouverneur ou gouvernante. Autre indice de changement, en 1803, on proposa d'engager deux veilleuses pour garder les malades la nuit ${ }^{64}$; et en 1805 , on décida d'en choisir deux pour les chambres des femmes malades et deux pour les hommes malades, la mortalité étant plus grande cette année-là, aux dires d'Abraham Joly ${ }^{65}$. Une étude reste encore à effectuer sur le rôle joué par ce personnel soignant et son évolution ${ }^{66}$.

Les médecins et les chirurgiens, comme on l'a vu plus haut, étaient présents à l'Hôpital dès sa création ou presque: toutefois, l'institution ne représentait pas un passage obligatoire pour mener à bien une carrière médicale. A l'époque qui nous intéresse, trois de chaque corps étaient employés par l'institution: l'un d'eux était chargé des malades de la «Maison», autrement dit des hospitalisés, et les deux autres avaient pour tâche de soigner les assistés de l'Hôpital à leur domicile, sur la demande des directeurs. En effet, chaque procureur dressait une liste des gens à soigner, dans le quartier dont il était responsable, et envoyait un médecin ou un chirurgien, selon la nature de la maladie; il est à relever que c'était également ces procureurs qui demandaient l'hospitalisation d'un malade, sans l'avis du médecin ou du chirurgien, du moins explicite. Cependant, en 1795, trois patients furent envoyés directement par le médecin, sans passer par un administrateur. 
Ainsi, si l'on en croit le peu de place accordée aux médecins et chirurgiens, et le silence des sources à leur propos, on serait en droit de déduire que leur influence médicale sur l'institution fut bien maigre et inconsistante. Toutefois, par des moyens détournés, on peut constater que leur rôle était plus important qu'il n'y paraît et dépendait de leur personnalité.

En effet, s'il est vrai que du point de vue institutionnel et administratif, ils n'avaient pas de grandes charges à assumer, si ce n'était de soigner des malades quand on le leur demandait, il n'en reste pas moins que certains d'entre eux eurent certainement une grande influence et distribuèrent les meilleurs soins possibles à leur malades. Mon propos ne sera pas de retracer ici la vie de tous les médecins et chirurgiens de l'Hôpital, cependant il me semble nécessaire de parler de deux figures appartenant à l'élite médicale genevoise, l'un chirurgien, Louis Jurine et l'autre médecin, Louis Odier. Jurine (1751-1819) fit ses études à Paris et fut admis à la maîtrise de chirurgie en 1773, à Genève ${ }^{67}$. Il devint chirurgien de l'Hôpital en $1781^{68}$ et resta chirurgien de la «Maison» jusqu'en 1786, date à laquelle les procureurs demandèrent aux médecin et chirurgien de l'Hôpital d'effectuer leur visite quotidienne ensemble. La charge sembla trop lourde à Jurine qui se vit dans l'obligation de donner sa démission à cause de l'étendue et de la nature de sa pratique ${ }^{69}$; nous n'avons malheureusement pas plus de détails sur sa pratique privée. Toutefois, Jurine proposa de rester chirurgien consultant de l'Hôpital et demeura par conséquent concerné par ce qui s'y passait. Après son passage hospitalier, Jurine devint membre correspondant de l'Institut de France, écrivit de nombreux travaux de médecine, de chirurgie et de sciences naturelles, et reçut en 1812 la moitié du premier prix d'un concours organisé par Napoléon, pour un mémoire qu'il avait écrit sur le croup ${ }^{70}$. Comme on le voit, c'était un homme d'une envergure internationale et il fit profiter l'institution hospitalière de son grand talent; en effet, demeurant consultant de l'établissement, il était appelé lors d'opérations délicates pour venir en aide à son successeur. D'autre part, il créa une maternité à Genève, favorisant ainsi la prise en charge médicale de l'accouchement ${ }^{71}$. Louis Odier (1748-1817) obtint son doctorat en médecine à Edimbourg, en 1770, et compléta ses études à Londres et à Paris ${ }^{72}$. En 1772, il acquit une grande expérience hospitalière dans la capitale britannique, à l'hôpital Saint-Thomas ${ }^{73}$, dans lequel il profita également de suivre des leçons d'anatomie et de dissection. C'est lui qui importa l'invention de Jenner en 1800 et grâce à lui et d'autres collègues, dont Jurine, Genève devint le centre de la diffusion de la vaccination sur le continent; membre de l'Institut de France, il participa à 
diverses sociétés médicales ${ }^{74}$. Or Louis Odier fut médecin de l'Hôpital pendant cinq ans, à son retour d'Angleterre ${ }^{75}$; si, comme Jurine, il fut extrêmement apprécié par les directeurs et contribua à la pratique d'une bonne médecine hospitalière, on peut toutefois s'étonner qu'Odier n'ait pas contribué à accélérer le processus de médicalisation de l'institution.

Ajoutons encore que la plupart des médecins genevois participaient activement à la Société de médecine ${ }^{76}$ et se réunissaient fréquemment et régulièrement pour discuter de cas qui les préoccupaient. Ainsi, non seulement les médecins de l'Hôpital avaient l'occasion de faire part de leurs difficultés, mais ils bénéficiaient également de l'expérience de leurs collègues. On lisait également, au sein de cette société, des traités de confrères étrangers de telle sorte que les médecins genevois bénéficiaient d'une grande ouverture sur la médecine européenne. On peut dire qu'à cette époque, les médecins se formaient à l'extérieur de l'Hôpital et faisaient activement profiter l'institution de leur savoir.

En revanche, certains restaient quelque peu imperméables à cette stimulation extérieure; c'est ainsi que Charles-Guillaume Dunant (1744-1808), qui fut médecin à l'Hôpital de 1789 jusqu'à sa mort, en 1808, après avoir terminé ses études à Montpellier, en 1772, et avoir été agrégé à Genève la même année, se distingua par son esprit routinier et peu novateur. En effet, en 1803, Jean Peschier (1774-1831) offrit aux directeurs ses services pour soigner les aliénés de l'Hôpital; en cela, il s'inscrivait dans le courant européen de ce tournant du siècle, qui portait un intérêt particulier pour la médecine des aliénés; le président de l'institution en fit part à Dunant, qui répondit qu'il voulait exercer ses fonctions tout seul, qu'il n'avait besoin de personne et qu'il demanderait de l'aide quand il en aurait besoin; d'autre part, selon son expérience, il était convaincu que les aliénés de l'Hôpital ne gagneraient rien à bénéficier de soins plus assidus ni à recevoir d'autres remèdes ${ }^{77}$; Peschier fut donc remercié et éconduit. On dut alors attendre la mort de Dunant pour engager Antonin Aubert, ce qui fut clairement dit d'ailleurs : le directeur «Trembley propose que l'on arrête que dans le cas où le médecin actuel de la maison viendroit à quitter, l'on séparera la maison des aliénés du reste de l'hopital et qu'elle aura un medecin particulier» ${ }^{78}$. En outre, Auguste Delarive, qui était déjà spécialisé dans le domaine des maladies mentales, notamment après avoir travaillé dans un asile moderne de Londres ${ }^{79}$, avait accepté de faire des visites bi-hebdomadaires aux aliénés de l'Hôpital, mais on dut également attendre la mort de Dunant ${ }^{80}$; Antonin Aubert fut finalement élu en $1808^{81}$. 
Comme on le voit, l'Hôpital ne laissant pas une place importante à la fonction de médecin ou de chirurgien, il était tributaire de la personnalité et du rayonnement de ces derniers.

\section{Conclusion}

Quelques remarques sur les indices de médicalisation de l'Hôpital général genevois me serviront de conclusion.

La responsabilité de l'institution envers les malades du territoire était présente dès sa création, avec notamment l'octroi des services d'un chirurgien et d'un médecin. Cette place laissée aux malades resta constante et se fit plus importante à la fin du XVIIIe siècle par le fait que l'on augmenta la capacité d'accueil des malades au sein de l'Hôpital. Cela représente à mes yeux un indice de médicalisation très important, car il révèle un changement de mentalité des administrateurs qui admettaient la fonction médicale de leur établissement.

L'amélioration des soins est moins visible, mais cela est en partie dû au fait que l'évolution du personnel médical n'a pas encore été étudiée de près. A première vue, on constate qu'à la fin de l'Ancien Régime, les directeurs accordent une certaine importance à la qualité du travail des gouverneurs, toutefois, il semblerait que leurs exigences ne furent remplies que vers le milieu du XIXe siècle ${ }^{82}$. Cependant, on peut tout de même constater une amélioration passive des soins ou plutôt une meilleure qualité de vie des malades au sein de l'institution dans le fait qu'on les déplace, en 1789, dans des chambres plus spacieuses et mieux aérées, et dans la volonté de les répartir selon la nature de leur affection. En outre, le désir des administrateurs d'employer au service de l'Hôpital des médecins et chirurgiens de grande envergure signifie évidemment une préoccupation importante concernant les soins accordés aux malades.

Si l'on considère maintenant l'Hôpital comme un lieu de formation, on en est pour le moment dans le domaine du pointillisme. En effet, dans l'état actuel des recherches, rien ne laisse penser que l'institution genevoise était un haut lieu de formation, ni médical, ni chirurgical. Cependant, il n'en reste pas moins qu'en 1746, comme on l'a vu plus haut, des dissections étaient effectuées pour améliorer l'apprentissage des sages-femmes. D'autre part, au début du XVIIIe siècle, une chambre anatomique fut installée dans une dépendance afin de donner des cours publics pour les chirurgiens. Enfin, au 
terme du XVIIIe siècle, l'hôpital, dans son concept, commence à avoir l'image d'un lieu de formation ou de perfectionnement aux yeux de la Faculté ${ }^{83}$. Ainsi, en 1791, G. B. Goty n'eut plus besoin de montrer des lettres d'apprentissage (dans une pratique privée) pour obtenir le droit de demander sa maîtrise, la Faculté considérant que, vu le développement des hôpitaux, une attestation hospitalière était équivalente ${ }^{84}$; en outre, Jérémie Macaire fut admis à sa maîtrise, malgré des examens très médiocres, à condition de suivre les traitements chirurgicaux qui étaient effectués à l'Hôpital ${ }^{85}$, pour compléter sa formation.

Quant à la distinction entre les motivations sociales, économiques ou médicales de soigner des malades, une évolution s'opère également. En effet, l'Hôpital avait été créé pour répondre à la misère quelle qu'elle fut, comme on le voit bien dans la phrase mise en exergue au début du premier registre des délibérations des procureurs ${ }^{86}$. Et on ne pourra jamais déterminer si un pauvre malade était accepté à cause de sa pauvreté ou de sa maladie. Or, dès 1785 , on a des listes, très sommaires, de patients qui payèrent pour se faire hospitaliser; ce n'est donc pas leur pauvreté qui les poussa à venir, mais le désir de se faire soigner et le sentiment d'être médicalement mieux pris en charge à l'Hôpital qu'à leur domicile. De plus, les procureurs décidèrent dans les années 1790, de demander aux malades qui le pouvaient de payer pour leur hospitalisation. Il est évident que cela correspondait à un moment de grandes difficultés financières, et ils devaient trouver de l'argent par tous les moyens. Cependant, cela indique qu'ils ne voyaient plus leur institution comme un organisme uniquement social, mais comportant également une spécificité médicale.

Cette étude ne concerne que deux décennies de la fin du XVIIIe siècle. On y a relevé un certain niveau de médicalisation: les administrateurs ont la volonté de privilégier les malades au détriment des valides «inutiles»; de même, on ne distingue plus seulement entre valides et malades, mais on opère désormais des séparations parmi ces derniers. D'autre part, on commence à faire payer, à ceux qui le peuvent, les soins administrés dans leur institution. Les médecins et les chirurgiens qui travaillent pour l'établissement sont généralement d'un bon niveau et assurent ainsi des soins de qualité, faisant profiter l'Hôpital de leur savoir constamment renouvelé et alimenté au sein de la Société de médecine.

Toutefois, ces résultats ne seront pleinement significatifs que lorsqu'ils pourront être comparés avec d'autres données concernant les périodes antérieure et postérieure. Les chiffres donnés ici doivent être inclus dans une 
longue série pour être révélateurs d'une tendance. Il est en outre nécessaire de déterminer les indices de médicalisation préexistants pour évaluer la détermination des autorités, la rapidité de la modernisation et l'ampleur de l'évolution, les changements de mentalité, face à l'hôpital ou face à la maladie. L'étude des années qui suivirent cette période est indispensable pour mesurer l'impact qu'eurent ces deux décennies sur la spécificité médicale de l'hôpital, sur le rôle croissant joué par le corps médical au sein de l'institution et même de la cité.

Notes

1 Sur la médicalisation de la société en général, voir Médicalisation de la société française, 1770-1850, édité par J. P. Goubert, Waterloo-Ontario, 1982; Jacques Léonard, Les médecins de l'Ouest au XIXe siècle, Thèse de l'Université de Paris IV, 1976, notamment t. 1 p. 75 à $120 ;$ D. Porter et R. Porter, Patient's progress. Doctors and Doctoring in Eighteenth-Century England, Cambridge, Polity Press 1989; Matthew Ramsey, Professionnal and popular medicine in France, 1770-1830. The social world of medical practice, Cambridge, University Press, 1988; R. H. Shryock, Histoire de la médecine moderne, Paris, Colin, 1956; Vincent Barras, «Pratique de la santé et hygiène publique», à paraître dans les Actes du colloque sur le Libéralisme genevois, Genève, 1992, Mémoires et Documents de la Société d'Histoire et d'Archéologie genevoise. Sur la médicalisation des hôpitaux, voir Colin Jones, The charitable imperative; hospitals and nursing in Ancien Régime and revolutionary France, Wellcome Institute Series in the History of Medicine, Londres/New York, 1989; Lindsay Granshaw, Roy Porter, The hospital in history, Wellcome Institute Series in the History of Medicine, 1989; Michel Foucault, Naissance de la clinique, Paris, Presses universitaires de France, 1963; Michel Foucault, Les machines à guérir; aux origines de l'hôpital moderne, Bruxelles, P. Mardage, 1977; E. H. Ackerknecht, La médecine hospitalière à Paris (17941848), Paris, Payot, 1986.

2 O. Faure, O. Dessertines, Populations hospitalisées dans la région lyonnaise au XIXe siècle et $X X e$ siècle, Villeurbanne, Centre national de la recherche scientifique, 1991, p. 6.

3 O. Keel, «La place et la fonction des modèles étrangers dans la constitution de la problématique hospitalière de l'Ecole de Paris» in History and Philosophy of the Life Sciences, vol. 6, no 1,1984 , p. 50 .

4 O. Keel, «La problématique de la clinique en France et à l'étranger, de la fin du XVIIIe siècle à la période de la Restauration», in Bulletin canadien d'histoire de la médecine, vol.2, no 2 , hiver 1985 , p. 193.

5 Ibid., p. 193.

6 C. Jones, The charitable imperative; hospitals and nursing in Ancien Régime and revolutionary France, Wellcome Institute Series in the History of Medecine, Londres/New York, 1989, p. 15.

7 C. Jones, ibid., p. 16; O. Faure, Genèse de l'hôpital moderne: les hospices civils de Lyon de 1802 à 1845, Lyon, Presses universitaires de Lyon, 1982, p. 39. 
8 Muriel Joerger, «La structure hospitalière de la France sous l'Ancien Régime», in Annales, 32 e année, no 5, septembre-octobre 1977, p. 1031.

9 C. Jones, ibid., p. 10.

10 Muriel Joerger, ibid., p. 1027.

11 Sandra Cavallo, «Charity, power and patronage in 18th century Italian hospital: the case of Turin» in Lindsay Granshaw, Roy Porter, The hospital in history, Wellcome Institute Series in the History of Medicine, 1989, p. 96 et suiv.

12 Eduard Seidler, «An historical survey of children's hospital» in ibid., p. 9, et 81 et suiv.; cet auteur s'appuie sur le développement des hôpitaux pour enfants, pour mettre à jour cette rupture.

13 John Henderson, «The hospital of Late-Medieval and Renaissance Florence : a preliminary survey» in ibid., p. 82.

14 Voir Colin Jones, The charitable imperative; hospital and nursing in Ancien Régime and revolutionary France, Wellcome Institute Series, 1989, p. 55 et suiv.

15 Guérir.

16 AEG, (pour Archives d'Etat de Genève) Arch. hosp. Aa 1, fol.1, 12.2.1542. Les archives hospitalières de l'Hôpital général de Genève forment un corpus de sources très complet, comprenant notamment les registres des délibérations des procureurs ( $\mathrm{Aa}$ ou $\mathrm{Ba}$ ), les registres d'entrées et sorties des pensionnaires $(\mathrm{Fa})$, les inventaires (Af), qui constituent les principales sources sur lesquelles est basée cette étude; ces archives se trouvent aux Archives d'Etat de Genève.

17 Le syndic est tenu d'assister à toutes les réunions des procureurs depuis 1552 déjà (AEG, Arch. hosp., Aa 2, fol.3, règlement du 13.3.1552).

18 Voir Eugène Olivier, Médecine et Santé dans le Pays de Vaud au XVIIIe siècle, Lausanne, Concorde, 1939, tome second, p. 780 à 791.

19 Léon Gautier, La médecine à Genève jusqu'à la fin du XVIIIe siècle, Genève 1906, MDG, p. 42 .

20 Passage cité par L. Fornara et B. Roth, «Un bâtiment neuf pour des ambitions nouvelles. La construction de l'Hôpital Général de Genève, de 1707 à 1712», in Sauver l'âme, nourrir le corps. De l'Hôpital Général à l'Hospice Général de Genève (1535-1985), publié par B. Lescaze, Genève, Hospice général, 1985, p. 182.

21 AEG, Arch. hosp., Ad 6, 22 et 26 juin 1746.

22 AEG, Arch. hosp., Ad 6, 13 février 1746.

23 Anne-Marie Barras-Dorsaz, «Un mode de répression genevois aux XVIIe et XVIIIe siècles: la maison de discipline» in Sauver l'âme, nourrir le corps, cité ci-dessus, p. 77 à 112.

24 Voir également sur ce sujet Vincent Barras, «Fers, bains et remèdes: «La maison des allienez», dans la Revue médicale de la Suisse romande, no 109, 1989, p. 999 à 1004.

25 Sur ce sujet, voir L. Fornara-B. Roth, ibid., p. 192-193.

26 Relevons que l'installation de lits de fer dans les années 1770 était relativement précoce si l'on se réfère aux hôpitaux parisiens, par exemple, qui ont passé des lits de bois aux lits de fer à partir de 1801 (voir Lydie Boulle, «La médicalisation des hôpitaux parisiens dans la première moitié du XIXe siècle», in Médicalisation de la société française, 1770-1830, édité par J. P.Goubert, Waterloo, Ontario, 1982, p. 38.

27 AEG, Arch. hosp., Af 4, inventaire de l'Hôpital général, 1772-1773. 
AEG, Arch. hosp., Aa 108, 24 mai 1789, p. 249-250.

29 AEG, Arch. hosp., Aa 108, 24 mai 1789, p. 250.

30 AEG, Arch. hosp., Aa 108, p. 348, 19 mai 1790.

31 AEG, Arch. hosp., Af 4, inventaire de 1772-1773.

32 AEG, Arch. hosp., Aa 108, p.52, 17 mai 1786.

33 AEG, Arch. hosp., Ab 1, p. 72, 20 mai 1799.

34 Ces dates sont déterminées par les inventaires des archives hospitalières.

35 AEG, Arch. hosp., Af 5, inventaire de 1825.

36 AEG, Arch. hosp., Aa 105, p.439, 4 janvier 1771.

37 Le Petit Hôpital était une dépendance de l'Hôpital général et se trouvait également au Bourg-de-Four, en face de ce dernier.

38 Voir L.Gautier, op. cit., p. 353.

39 AEG, Arch. hosp., Af 3.

40 AEG, Arch. hosp., Af 5.

41 Les chiffres et les estimations données dans ce chapitre sont basés sur l'analyse détaillée des années 1785,1790 et 1795. Bien entendu, ces chiffres ne sont donnés qu'à titre indicatif, et ne permettent pas de tirer des conclusions concernant une évolution quelconque, mais donnent toutefois des indications ponctuelles.

42 Sur lequel voir W. Zurbuchen, «Une grande figure oubliée: Abraham Joly», in Sauver l'âme, nourrir le corps, op.cit., p. 327 à 331 . Soulignons au passage que Joly avait été directeur de l'Hôpital, puis hospitalier; cependant, avant d'occuper ces charges administratives, il avait été médecin.

43 AEG, Arch. hosp., Aa 108, p. 212, 7 janvier 1789.

44 AEG, Arch. hosp., Aa 107, p. 428, 25 avril 1784, p. 465, 19 décembre 1784.

45 Voir O. Faure et D. Dessertines, Populations hospitalisées dans la région lyonnaise aux XIXe et XXe siècles, Villeurbanne, 1991, p. 32 .

46 AEG, Arch. hosp., Fa 15, 20 août 1795.

47 AEG, Arch. hosp., Fa 15, 6 décembre 1790.

48 O. Faure et D. Dessertines, op. cit., p. 91.

49 AEG, Arch. hosp., Fa 15, p. 78, 89, 104 et 141.

50 AEG, Arch. hosp., Fa 15, p. 10, 3 juillet 1785.

51 AEG, Arch. hosp., Fa 15, p.11, 13 juillet 1785.

52 Dans le dictionnaire de Panckoucke, l'auteur de la rubrique ajoute que le terme d'asthme est souvent confondu avec «dyspnée», qui est toujours symptomatique d'une autre affection, comme une maladie organique du cœur ou des gros vaisseaux (voir Dictionnaire des sciences médicales, édité par C.L.F. Panckoucke, Paris, 1812, tome 2).

53 Ce terme était défini comme la suite ou un mode particulier de développement de diverses affections morbides, telles que l'inflammation, l'hémorragie, les scrofules, etc. ... Panckoucke, ibid., tome 42, p. 24.

54 Sur la difficulté du diagnostic rétroactif, voir Jean-Pierre Peter, «Malades et maladies à la fin du XVIIIe siècle», dans Médecins, climat et épidémies à la fin du XVIIIe, publié par J. P. Desaive, Paris, La Haye, Mouton, 1972, p. 146 et suiv., et Mirko D. Grmek, Les maladies à l'aube de la civilisation occidentale, Paris, Payot, 1983, p. 19 à 21.

55 Lydie Boulle, op.cit., p. 34. 
56 Le Livre des Morts enregistrait toutes les personnes décédées à Genève, avec leurs noms, leur âge, parfois leur profession, leur domicile et surtout la cause de leur mort; il était tenu par le visiteur des morts qui n'était autre qu'un chirurgien de l'Hôpital.

57 Les termes de «interne» et de «dermatologique» ne figurent bien évidemment pas dans les sources. Sous le vocable «interne» figurent les fièvres, hydropisie, phtisie, paralysie, tétanos, rougeole, affection de poitrine, dysenterie, cachexie, obstruction du bas ventre, malaise.

58 Parmi lesquelles, ulcère, fausse rache, dartre, engelures, vermine, abcès, érésipèle, et galle, surtout en 1795, qui en compta 24 cas.

59 Il s'agissait le plus souvent de femmes célibataires ou abandonnées.

60 Comme Madelaine Dalgue, femme de Jean-Pierre, qui resta 12 jours à l'Hôpital lors de son accouchement (AEG; Arch. hosp., Fa 15, 27 mai 1795).

61 AEG, Arch. hosp., Ab 2, p. 180 et 182, 8 et 22 septembre 1807. Ajoutons que Louis Jurine était chirurgien de l'Hôpital à la fin du siècle précédent.

62 O. Faure et D. Dessertines, op.cit., p. 44.

63 AEG, Arch. hosp., Aa 109, p. 381, 22 juin 1796.

64 AEG, Arch. hosp., Ab 2, p.2, 2 août 1802.

65 AEG, Arch. hosp., Ab 2, p. 84, 19 mai 1805.

66 Joëlle Droux est en train de faire une thèse sur ce sujet, à l'Université de Genève, département d'histoire.

67 AEG, Santé F 1, p. 270-271, 14 décembre 1772, 7 avril et 23 juin 1773.

68 AEG, Arch. hosp., Aa 107, p. 281 et 285, 23 septembre et 28 octobre 1781.

69 AEG, Arch. hosp., Aa 108, p. 45-46, 26 mars 1786.

70 Voir, M. Folman, «Le prix Napoléon et Louis Jurine», in La Revue médicale de la Suisse romande, 25 janvier 1948, 68e année, no 1; Albert Montet, Dictionnaire biographique des Genevois et des Vaudois, Lausanne, Bridel, 1878, p.17-18.

71 Voir note 52. Louis Jurine fait actuellement l'objet d'une recherche effectuée par Vincent Barras et l'auteur.

72 E. Thomas, La médecine à Genève au début du XIXe siècle, Publication des Archives de la Société Médicale de Genève, Bâle, 1937, p. 12-13; «Notice biographique sur feu Monsieur le Professeur Odier», publiée dans la Bibliothèque Universelle, vol.41, 1817.

73 Cet hôpital pouvait contenir 450 malades; sa pharmacie avait retenu l'attention du Docteur Alex. Flajani au cours de son voyage. Voir Louis Odier, «Extraits d'un ouvrage du Dr Alex. Flajani, de Rome (sur les établissements publics relatifs à la médecine)», publié dans la Bibliothèque Britannique, Genève, 1811, p. 67-68.

74 Voir M. A. Barblan, «Journalisme médical et échanges intellectuels au tournant du XVIIIe siècle : le cas de la Bibliothèque Britannique (1776-1815)», Archives des Sciences 30 (3), 1977, p. 296 et p. 378 à 380 qui donnent les références de la correspondance de Louis Odier concernant la vaccination.

75 AEG, Arch. hosp., Aa 107, p. 253, 10 janvier 1781.

76 Sur laquelle voir J. Olivier, «Les sociétés genevoises de médecine» in Revue médicale de la Suisse romande 57, 1937, p. 664 à 686 . Une recherche est actuellement en cours sur ces sociétés, avec notamment une problématique concernant les rapports existants entre médecins et chirurgiens, recherche effectuée par O. Keel, V. Barras et l'auteur.

77 AEG, Arch. hosp., Ab 2, p. 9, 18 septembre 1803. 
78 AEG, Arch. hosp., Ab 2, p. 190, 10 novembre 1807.

79 «Notice biographique sur Monsieur le Professeur G. de La Rive», dans Bibliothèque Universelle, mars 1834.

80 AEG, Arch. hosp., Ab 2, p. 192, 8 décembre 1807.

81 AEG, Arch. hosp., Ab 2, p. 209 et 210, 19 et 26 avril 1808.

82 Ce renseignement nous a été aimablement fourni par Joëlle Droux.

83 Il ne s'agit pas ici de faculté académique, mais de l'ensemble des médecins, chirurgiens et apothicaires de la ville.

84 AEG, Santé F 1, p. 304, 8 avril 1791, p. 306, 6 juillet 1791.

85 AEG, Santé F 1, p. 299-300, 23 septembre 1790.

86 Voir la citation de la page 2 .

Micheline Louis-Courvoisier

Institut Louis Geantet

d'histoire de la médecine

Case Postale

1211 Genève 4 